\title{
Measurement of the psychosis continuum: Modelling the frequency and distress of subclinical psychotic experiences
}

\author{
Mark Shevlin $^{a}$, David Boyda ${ }^{a}$, James Houston $^{\mathrm{a}}$ \& Jamie Murphy ${ }^{\mathrm{a}}$ \\ a \\ School of Psychology and Psychology Research Institute, University of \\ Ulster, Magee, Northern Ireland Published online: 04 Aug 2014
}

\author{
Measurement of the psychosis continuum: Modelling the frequency \\ and distress of subclinical psychotic experiences \\ Mark Shevlin*, David Boyda, James Houston and Jamie Murphy \\ School of Psychology and Psychology Research Institute, University of Ulster, \\ Magee, Northern Ireland
}

(Received 25 August 2013; accepted 2 June 2014)

Objective: Dimensional models of psychosis symptom frequency at clinical levels are representative of symptom dimensionality that is inclusive of distress. However, factor models of psychotic-like experiences, or subclinical symptomatology, in the general population have only ever been estimated using information on the frequency of occurrence. To ascertain whether dimensional representations of psychosis at subclinical levels are reflective of clinical manifestations of psychosis, factor models must utilise data that permits the measurement of both frequency and distress of psychosis experiences. Method: Psychotic-like experiences were assessed in a nonclinical sample $(\mathrm{N}=462)$ using the 20 positive items from the CAPE42, which is a self-report questionnaire of psychotic experiences. For each item of the CAPE the frequency and distress ratings were recoded to form composite scores. Seven factor analytic models were specified and tested using confirmatory factor analysis. Results: The five-factor model of Wigman et al. (hallucinations, paranoia, grandiosity, delusions and paranormal beliefs factors) represented the best fitting model for both frequency and composite data.

Conclusions: The findings constitute further evidence for a continuum of psychosis within the general population. Future analyses, aimed at delineating the dimensionality of psychosis, must advance towards the inclusion of distress as a central and necessary adjunct to measurement.

Keywords: psychosis; continuum; distress; confirmatory factor analysis

\section{Introduction}

It has been hypothesised that the psychosis phenotype is continuous in nature and produces a skewed distribution of symptom severity in the general population (Ahmed, Buckley, \& Mabe, 2012). The upper end of this distribution is hypothesised to represent clinical psychosis with symptoms that are distressing, debilitating and result in the need for treatment, while the lower end is hypothesised to represent the absence of symptoms or "normality". It is proposed that subclinical psychosis lies 
somewhere between these extremes and represents individuals who (a) experience one or more psychotic symptoms but do not meet the criteria for a diagnosis of a psychotic disorder or (b) experience psychotic-like experiences (PLEs) that are phenomenologically similar to psychotic symptoms but that may or may not be distressing in nature. These experiences have been labelled "subclinical psychotic experiences" and "subclinical psychotic symptoms" and they have been shown to have a prevalence in the general population of approximately $8 \%$ and $4 \%$, respectively.

It has been proposed that the assessment of subclinical psychotic experiences and symptoms may be important as they may constitute risk factors for transition to clinical psychotic disorder (Parker \& Lewis, 2006; Poulton et al., 2000). However, to date, existing self-report measures of psychosis have generally asked respondents to only rate the frequency of particular psychotic symptoms but have not asked respondents about the distress associated with these symptoms. This frequencyonly/distress-absent measurement of psychosis potentially compromises judgements about the comparability of subclinical and clinical manifestations of psychotic experience. Without a measure of distress it is difficult to establish whether subclinical experiences are merely indicative of variations or eccentricities in personality (e.g. schizotypal personality) or whether they predict subclinical psychotic symptoms. It would seem plausible to assume therefore that a measure that combined information on the frequency of symptom occurrence with the associated distress of the symptom would be better placed to assess the "true nature" of a person's experiences and in so doing, their position along the psychosis continuum.

Recent advances in the conceptualisation and measurement of psychosis have suggested that the psychosis phenotype may be both dimensional and continuous in nature. Factor-analytic studies, for example, have revealed replicable dimensional structures (i.e. distinct groups of psychosis symptoms or experiences that are likely to co-occur) within both clinical and nonclinical or subclinical populations (Boyda, Shevlin, Mallett, Murphy, \& Houston, 2013; Reininghaus, Priebe, \& Bentall, 2012). More specifically, research has suggested that positive symptoms are best explained by two (hallucinations and delusions: Mellers, Sham, Jones, Toone, \& Murray, 1996) or three (hallucinations, Schneiderian first-rank delusions, and paranoid delusions: Murphy, Shevlin, Adamson, \& Houston, 2010) correlated dimensions.

More complex structures have also been reported; however, these have been identified mainly by studies that used the positive items from the Community Assessment of Psychiatric Experiences questionnaire (CAPE; Stefanis et al., 2002). For example, Yung et al. (2009) using a community sample of adolescents $(n=875)$ conducted a maximum likelihood factor analysis with direct oblimin rotation and reported a 4-factor solution identifying the dimensions: bizarre experiences, perceptual abnormalities, persecutory ideation, and magical thinking. However, this model contained a small number of cross-factor loadings. Barragan, Laurens, 
Navarro, and Obiols (2011) used the Spanish version of the CAPE with a sample of 777 adolescents. A principal components analysis identified a 4-component structure. The dimensions in this analysis were persecutory ideation, grandiose thinking, first rank/ hallucinatory experiences and self-referential thinking. Recently, Wigman et al. (2011) employed exploratory and confirmatory analyses to investigate the dimensional structure of psychosis using a large sample of adolescents $(n=$ 5422). They reported an optimal solution including five factors: hallucinations, paranoia, grandiosity, delusions and paranormal beliefs.

It is proposed, therefore, that any measurement of a hypothesised psychosis continuum should, where possible, utilise assessment tools that can adequately (i) quantify the frequency of symptoms/psychotic-like experiences, (ii) assess if there is associated distress with these symptoms and psychotic-like experiences, and (iii) ensure that the scores are multidimensional and consistent with existing clinical research findings. Therefore, the main aim of this study was to assess the viability of a scoring system for the CAPE that combined the frequency of symptom experiences and associated distress caused by these experiences using "composite" scores. This method involved combining information on frequency and distress at the item level rather than separate scores of overall frequency and overall distress. This revised method of scoring permitted the use of composite scores at the level of the subscale and total scale, where to date, the distress items from the CAPE have traditionally been used for a separate overall distress scale score. The viability of this novel scoring system will be assessed by determining if the resultant composite scores produce factors consistent with previous research findings (i.e. models based on frequency scores alone). Based on previously reported fit statistics for alternative dimensional models of psychosis it was tentatively predicted that a multidimensional model would be the best fitting model and that models with a greater number of dimensions would produce greater fit (e.g. Wigman et al., 2011). However, while it was expected that the current distress encoded psychosis items would mimic clinical dimensions of psychosis it was not possible to predict with confidence which of the six multidimensional models would perform best among this nonclinical sample.

\section{Method Participants and sampling}

A convenience sample of undergraduate students from two UK universities was used (University A, $\mathrm{N}=272,60$ (22\%) males; University B, $\mathrm{N}=190,57$ (30\%) males). The final sample consisted of 462 participants, 117 males $(25 \%)$ and 345 females $(75 \%)$. The mean age of the sample was 21.2 years $(\mathrm{SD}=5.4)$ with an age range from 18 to 34 years. The majority described themselves as single $(75.5 \%)$ or cohabiting (19.0\%) with the remainder describing themselves as either married, divorced or separated. Moreover, 91.5\% described themselves as "White", and a large proportion reported living in an urban area $(68.0 \%)$. Less than half of the sample $(42.6 \%)$ reported ever having used cannabis. 
Measures

The Community Assessment for Psychic Experiences (CAPE: Stefanis et al., 2002)

Psychotic-like experiences were assessed using the 20 positive items of the CAPE 42. The CAPE-42 is a 42-item self-report questionnaire, derived from the Peters et al. Delusions Inventory (PDI-21; Peters, Joseph, \& Garety, 1999), measuring positive (20 items) and negative (14) psychotic symptoms and depressive (8 items) symptoms on a two-dimensional scale. The first dimension measures the frequency of symptoms on a four-point scale of "never" $=1$, "sometimes" $=2$, "often" $=3$ and "nearly always" $=4$, and the second dimension measures the degree of distress caused by the experience: "not distressed" $=1$, "a bit distressed" $=2$, "quite distressed" = 3 and "very distressed" = 4. Ratings of distress for each item are not permitted when the item was not endorsed (i.e. "never"). The current study utilised only the 20 positive items which assess symptoms related to referential thinking, magical ideation, hallucinations and suspiciousness. The CAPE has been evidenced to demonstrate good internal consistency with a Cronbach's alpha of $r=0.83$ and it has been administered to both clinical and nonclinical populations (Armando et al., 2010; Stefanis et al., 2002; Yung et al., 2006, 2009).

\section{Data preparation and analyses}

For each CAPE item the frequency and distress scores were combined to produce a new variable with three levels. These were ordered levels representing (1) nonendorsement of item, (2) endorsement with no associated distress, and (3) endorsement with associated distress. Specifically, if the original frequency was rated "never", the new variable was coded " 1 ", and if any other frequency was endorsed ("sometimes", "often", or "nearly always") and the distress was scored "not at all distressed" the new variable was coded " 2 ". The third level of the new variable represented those responses that were rated greater than or equal to "sometimes" on the frequency scale and also greater than or equal to "a little distressed" on the distress scale. This produced 20 item-level composite scores with possible values ranging from 1 to 3 and total scaled scores with a possible range from 20 to 60, with higher scores indicating higher levels of subclinical psychotic symptoms. The resultant scoring method provided a combined measure of both frequency and distress.

Seven factor-analytic models were specified and tested within a confirmatory factor analytic (CFA) framework using Mplus 6.00 (Muthén \& Muthén, 1998). The one-factor model tested whether all the scores could be explained by a single, global dimension of "positive symptoms". The two- and three-factor models were based on studies by Mellers et al. (1996) and Murphy et al. (2010) who used the Present State Examination (Wing, Cooper, \& Sartorius, 1974) and the Composite International Diagnostic Interview (WHO, 1990), respectively. These models dictated the structure of the two- and three-factor models based on the CAPE items. The remaining models were based on factor-analytic studies based on the CAPE. Two four-factor models by Yung et al. (2009) were tested; one that included cross-factor 
loadings and one with only the highest factor loadings, a "simple structure" model. A four-factor model by Barragan et al. (2011) and a five-factor model by Wigman et al. (2011) were also tested. The structure of the models is presented in Table 1.

The composite items were modelled as categorical variables using the robust weighted least squares estimator (WLSMV) based on the polychoric correlation matrix of latent continuous response variables. The WLSMV estimator is the most appropriate statistical treatment of categorical indicators in a CFA context (Brown, 2006). Other methods of analysis, such as maximum likelihood estimation, tend to produce incorrect standard errors, attenuate the relationships between observed variables and produce possible pseudo-factors (Brown, 2006). The WLSMV estimator has been shown to produce correct parameter estimates, standard errors and test statistics (Flora \& Curran, 2004). Goodness of fit for each model was assessed with a range of fit indices including the MLR chi-square (Muthén \& Muthén, 1998), the comparative fit index (CFI; Bentler, 1990), and the TuckerLewis Index (TLI; Tucker \& Lewis, 1973). A nonsignificant $\chi^{2}$ and values greater than .90 for the CFI and TLI were considered to reflect acceptable model fit. Additionally, the Root Mean Square Error of Approximation (RMSEA; Steiger, 1990) was reported, where a value less than .05 indicated close fit and values up to .08 indicated reasonable errors of approximation (Jöreskog \& Sörbom, 1993). For all models the factors were specified to be correlated and the error variances were uncorrelated.

Results

Descriptive statistics for the CAPE items are presented in Table 2.

Table 1. Item map of the alternative models of the positive items of the CAPE.

\begin{tabular}{|c|c|c|c|c|c|c|c|}
\hline Items & 1 Factor & $\begin{array}{l}\text { Mellers } \\
\text { et al. } \\
(1996)\end{array}$ & $\begin{array}{l}\text { Murphy } \\
\text { et al. } \\
(2010)\end{array}$ & $\begin{array}{l}\text { Yung } \\
\text { et al. } \\
(2009)\end{array}$ & $\begin{array}{l}\text { Yung } \\
\text { et al. } \\
(2009)\end{array}$ & $\begin{array}{l}\text { Barragan } \\
\text { et al. } \\
(2011)\end{array}$ & $\begin{array}{l}\text { Wigman } \\
\text { et al. } \\
(2011)\end{array}$ \\
\hline & & 2 Factor & 3 Factor & 4 Factor & 4 Factor & 44 Factor & 5 factor \\
\hline 1 & $\mathrm{P}$ & Delu & Pers & Pers & Pers & Pers & Pers \\
\hline 2 & $\mathrm{P}$ & Delu & Hall & Pers * & Pers & Self & Delu \\
\hline 3 & $\mathrm{P}$ & Delu & Pers & Pers & Pers & Pers & Pers \\
\hline 4 & $\mathrm{P}$ & Delu & Pers & Pers & Pers & Pers & Pers \\
\hline 5 & $\mathrm{P}$ & Delu & Pers & Pers & Pers & Pers & Pers \\
\hline 6 & $\mathrm{P}$ & Delu & Firs & Magi & Magi & Gran & Gran \\
\hline 7 & $\mathrm{P}$ & Delu & Firs & Magi * & Magi & Gran & Gran \\
\hline 8 & $\mathrm{P}$ & Delu & Hall & Magi & Magi & Firs & Para \\
\hline 9 & $\mathrm{P}$ & Delu & Firs & Biza & Biza & Self & Delu \\
\hline 10 & $\mathrm{P}$ & Delu & Firs & Magi & Magi & Firs & Para \\
\hline 11 & $\mathrm{P}$ & Delu & Pers & Pers & Pers & Pers & Pers \\
\hline 12 & $\mathrm{P}$ & Delu & Firs & Biza & Biza & Firs & Delu \\
\hline 13 & $\mathrm{P}$ & Delu & Firs & Biza & Biza & Firs & Delu \\
\hline
\end{tabular}




$\begin{array}{llllllll}14 & \text { P } & \text { Delu } & \text { Firs } & \text { Biza } & \text { Biza } & \text { Firs } & \text { Delu } \\ 15 & \text { P } & \text { Delu } & \text { Firs } & \text { Biza } & \text { Biza } & \text { Firs } & \text { Delu } \\ 16 & \text { P } & \text { Delu } & \text { Firs } & \text { Biza } & \text { Biza } & \text { Firs } & \text { Delu } \\ 17 & \text { P } & \text { Hall } & \text { Hall } & \text { Perc } & \text { Perc } & \text { Firs } & \text { Hall } \\ 18 & \text { P } & \text { Hall } & \text { Hall } & \text { Perc } & \text { Perc } & \text { Firs } & \text { Hall } \\ 19 & \text { P } & \text { Delu } & \text { Pers } & \text { Pers } & \text { Pers } & \text { Firs } & \text { Delu } \\ 20 & \text { P } & \text { Hall } & \text { Hall } & \text { Perc* } & \text { Perc } & \text { Firs } & \text { Hall }\end{array}$

Note: P, Positive Symptoms; Pers, Persecutory Ideation; Biza, Bizarre Experiences; Magi, Magical Thinking; Perc, Perceptual Abnormalities; Delu, Delusions; Gran, Grandiosity; Para, Paranormal Beliefs; Hall, Hallucinations; Self, Self Referential Thinking; Firs, First Rank Symptoms.

*Cross-factor loaded items.

The composite scoring method appeared to generate adequate variability of scores: the minimum and maximum values indicated that for half of the items the entire possible range of scores (0-3) was observed. The means for the items were generally low ranging from 1.11 to 2.48 . As expected, the distribution of scores were positively skewed.

The Wigman et al. (2011) five-factor model represented the best fitting model for both frequency and composite data (see Table 3). Although the chi-square statistics were statistically significant, this did not lead to the rejection of the models as the large sample size increased the power of the tests (Tanaka, 1987). All other fit statistics indicated acceptable model fit, and the Wigman model was significantly better for both frequency $\left(\chi^{2}=220.164, \mathrm{df}=160, \mathrm{p}=<0.00\right), \mathrm{CFI}=.97$, TFI=.97, RMSEA=.029) and the composite $\left(\chi^{2}=202.469, \mathrm{df}=160, \mathrm{p}=<0.00\right), \mathrm{CFI}=.98$, TFI=.98, RMSEA=.024) than the next best model (Barragan four-factor model). The standardised factor loadings and factor correlations for the Wigman et al. (2011) five-factor model are presented in Figure 1.

Cronbach's alpha was calculated for the total CAPE and for each of the subdimensions derived from the five0factor model: $\alpha=.82$ (Total), $\alpha=.53$ (Hallucinations), $\alpha=.62$ (Paranoia) and $\alpha=.53$ (Grandiosity), $\alpha=.71$ (Delusions), $\alpha$ $=.57$ (Paranormal). The intercorrelations for the five subdimensions were all statistically significant ranging from $\mathrm{r}=.20$ to .47 , all $\mathrm{p}=<.001$. The means and standard deviations for the positive items of the CAPE and its subdimensions derived

Table 2. Descriptive statistics for the CAPE items using a summed frequency and distress scoring method.

\begin{tabular}{lccccc}
\hline & Min & Max & Mean & Std. Deviation & Skew \\
\hline Double meaning & 1 & 3 & 2.18 & .803 & -.334 \\
Messages from TV & 1 & 3 & 1.35 & .614 & 1.540
\end{tabular}


M. Shevlin et al.

\begin{tabular}{lllllr} 
People not what they seem & 1 & 3 & 2.48 & .624 & -.79 \\
Being persecuted & 1 & 3 & 1.58 & .868 & .92 \\
Conspiracy & 1 & 3 & 1.28 & .668 & 2.08 \\
Being important & 1 & 3 & 1.64 & .718 & .65 \\
Being special & 1 & 3 & 1.54 & .678 & .86 \\
Telepathy & 1 & 3 & 1.40 & .624 & 1.30 \\
Influenced by devices & 1 & 3 & 1.56 & .781 & .94 \\
Voodoo & 1 & 3 & 1.47 & .757 & 1.22 \\
Odd looks & 1 & 3 & 1.80 & .899 & .40 \\
Thought withdrawal & 1 & 3 & 1.20 & .567 & 2.67 \\
Thought insertion & 1 & 3 & 1.31 & .696 & 1.93 \\
Thought broadcasting & 1 & 3 & 1.37 & .739 & 1.60 \\
Thought echo & 1 & 3 & 1.34 & .650 & 1.71 \\
External control & 1 & 3 & 1.22 & .592 & 2.51 \\
Verbal hallucinations & 1 & 3 & 1.20 & .574 & 5.80 \\
Voices conversing & 1 & 3 & 1.05 & .317 & 4.0 \\
Capgras & 1 & 3 & 1.11 & .450 & 3.82 \\
Visual hallucinations & 1 & 3 & 1.15 & .512 & 3.18 \\
Total Scale Score & 1 & 60 & 29.30 & 6.32 & 1.58 \\
\hline
\end{tabular}

Note: All estimates of skew were statistically significant $(\mathrm{p}<.05)$.

from the five-factor model were as follows: CAPE Total $(\mathrm{M}=29.19$, $\mathrm{SD}=6.32)$, Hallucinations $(M=3.41, S D=1.03)$, Paranoia $(M=9.31, S D=2.47)$, Grandiosity $(\mathrm{M}=3.18, \mathrm{SD}=1.15)$, Delusions $(\mathrm{M}=10.45, \mathrm{SD}=2.95)$, and Paranormal $(\mathrm{M}=$ $2.87, \mathrm{SD}=1.16)$.

\section{Discussion}

The main aim of this study was to assess the viability of a scoring system for the CAPE that combined the frequency of symptom experiences and the degree of distress caused by the symptoms using item-level composite scores. The composite scores were, as expected, positively skewed and the mean scores were generally low. The factor analyses of alternative models based on the composite scores produced fit statistics that were superior to those from analyses based on frequency ratings only. This suggested that subclinical psychotic symptoms not only shared phenomenological similarities with clinical psychotic symptoms, but also had a similar capacity to promote distress.

These findings may help improve our understanding of psychosis symptom expression within the general population. Recent advances in the conceptualisation and measurement of psychosis have suggested that the psychosis phenotype may be both multidimensional and continuous in nature. For example, factor-analytic studies have revealed replicable dimensional structures (i.e. distinct groups of psychosis symptoms or experiences that are likely to co-occur) within both clinical and 
nonclinical or subclinical populations, and latent class analyses have identified distinct, homogeneous groups of individuals who share similar psychosis symptom or

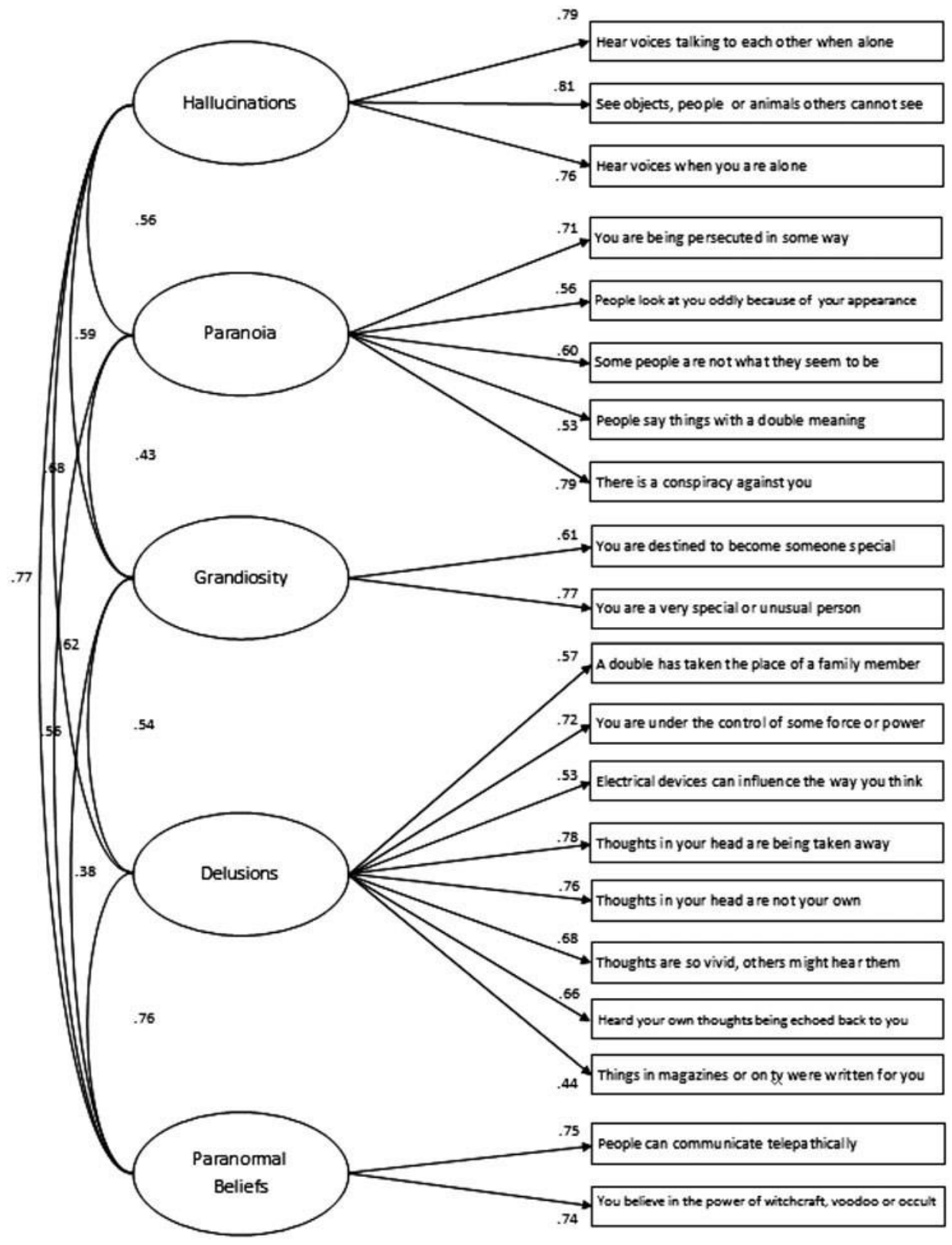

Figure 1. Path model with standardised factor loadings and factor correlations. 
experience profiles (i.e. groups of individuals characterised by distinct profiles of psychosis who are comparable to other groups of individuals in terms of the severity or probability of the occurrence of these symptoms or experiences).

While findings from these analyses have been presented as support for a continuum hypothesis of psychosis they have been successful only in so far as they have demonstrated the existence of a stable and continuous psychosis structure within the population. However, stability and consistency in the presentation or expression of psychosis across the clinical divide, while necessary if one is to speculate about the presence of a continuum, are not sufficient alone to fully justify a continuum-based representation of psychotic experience. Variation in distress along the continuum must also be considered.

Many studies assessing the dimensionality of psychosis have been conducted on patient populations; chronic psychosis sufferers in the exacerbation phase of their illness (Peralta \& Cuesta, 2001). However, the exacerbation phase is only one of several phases experienced by psychotic individuals and as a consequence research has also investigated the factor structure of the disorder at other phases of the illness. For example, several studies have examined the dimensional structure of psychosis at the stable phase of the illness (e.g. Malla, Norman, Williamson, Cortese, \& Diaz, 1993; Schuldberg, Quinlan, Morgenstern, \& Glazer, 1990), during partial remission (e.g. Goldman, Tandon, Liberzon, Goodson, \& Greden, 1991; Jackson, Burgess, Minas, \& Joshua, 1990), and also at full remission (e.g. Czobor \& Volavka, 1996; Nakaya, Suwa, Komahashi, \& Ohmori, 1999). While many researchers have reported stable and replicable factor structures, some have reported noticeable variability in the factor structure of the symptoms at different times of assessment.

However, factor analyses of psychosis symptomatology using clinical data inadvertently capture distress, given the health status of those providing the data (i.e. individuals diagnosed and in receipt of treatment because of distressing psychotic experiences or distress associated with psychotic experiences). Therefore, in these circumstances, resultant factor solutions reflect the structure of psychosis where distress is already inherent. Subclinical phenomena may not, in many cases, be distressing, however.

The continuum hypothesis posits that clinically defined psychosis is distinguishable from nonclinical or subclinical psychosis by virtue of its clinical relevance (i.e. its associated distress) and according to this hypothesis, subclinical expressions of psychosis are merely quantitatively different from more extreme phenotypic expressions (i.e. they differ in level/severity but not in form) and as such should also be indicative of distress but to a lesser extent. If the dimensionality of psychosis at the clinical/pathological level is different to that within the wider community then this would suggest that psychosis is qualitatively different within a clinical context. If this were to be the case then more traditional categorical/dichotomous perspectives might prove to be more appropriate conceptualisations of the phenomenon. However, if existing dimensional representations of subclinical psychosis, that are comparable with clinical 
representations, remain stable when they are estimated in conjunction with an associated measure of distress, then the continuum based interpretations previously attributed to stable factor structure findings alone (based on frequency of symptom occurrence only) may be strengthened. The factor structure combining the frequency of symptom experiences and the degree of distress caused by the symptoms using item level composite scores modelled in the current analysis seemed to achieve this stability.

There were some methodological limitations associated with this study that should be noted. While every effort was made in the current study to measure "true" subclinical psychotic experiences, the measurement of psychosis-like symptoms can be confounded by numerous factors such as respondents' misunderstanding the nature of the questions (e.g. question about hearing things others cannot being interpreted as a question about hearing ability) or normalising the experiences (e.g. 
M. Shevlin et al.

interpreting the paranoia question as relating to actual experienced threat/harm). In addition, it is not easy from lay interviews to distinguish reports of odd experiences from true psychotic experiences. Also, while self-report measures of psychotic experience may be accurate in clinical samples, they may be falsely denied in the general population due to the perceived stigma associated with such experiences. There were also some methodological limitations regarding the high representation of females in our sample. Finally, the findings would have been strengthened if outcome measures (help-seeking, social/occupational functioning, other existing psychological disorders, etc.) had been included.

To conclude, the present findings support a five-factor model of psychosis as described by Wigman et al. (2009) and supports a continuum model of psychosis that extends beyond the "disorder" construct and acknowledges the prevalence of these symptoms and experiences in the general population (Fanous, Gardner, Walsh, \& Kendler, 2001; Verdoux \& van Os, 2002). If, as has been suggested by the current findings, psychosis does exist along a continuum of symptom severity, then future analyses, aimed at delineating the dimensionality of the disorder, must advance towards the inclusion of distress as a central and necessary adjunct to measurement.

\section{References}

Ahmed, A.O., Buckley, P.F., \& Mabe, P.A. (2012). Latent structure of psychotic experiences in the general population. Acta Psychiatrica Scandinavica, 125, 54-65.

Armando, M., Nelson, B., Yung, A.R., Ross, M., Birchwood, M., Girardi, P., \& Nastro, P.F. (2010). Psychotic-like experiences and correlation with distress and depressive symptoms in a community sample of adolescents and young adults. Schizophrenia Research, 119, $258-265$.

Barragan, M., Laurens, K.R., Navarro, J.B., \& Obiols, J.E. (2011). Psychotic-like experiences and depressive symptoms in a community sample of adolescents. European Psychiatry, 26, 396-401.

Bentler, P. (1990). Comparative fit indexes in structural models. Psychological Bulletin, 107, 238-246.

Boyda, D., Shevlin, M., Mallett, J., Murphy, J., \& Houston, J. (2013). Confirmatory factor analysis of the schizotypal personality questionnaire - Brief: An alternative models approach. Psychosis: Psychological, Social and Integrative Approaches, 5, 187-196.

Cuesta, M.J., \& Peralta, V. (2001). Integrating psychopathological dimensions in functional psychoses: A hierarchical approach. Schizophrenia Research, 52, 215-229.

Czobor, P., \& Volavka, J. (1996). Dimensions of the brief psychiatric rating scale: An examination of stability during haloperidol treatment. Comprehensive Psychiatry, 37, 205-215.

Goldman, R.S., Tandon, R., Liberzon, I., Goodson, J., \& Greden, J.F. (1991). Stability of positive and negative symptom constructs during neuroleptic treatment in schizophrenia. Psychopathology, 24, 247-252.

Jackson, H.J., Burgess, P.M., Minas, I.H., \& Joshua, S.D. (1990). Psychometric properties of the manchester scale. Acta Psychiatrica Scandinavia, 81, 108-113.

Jöreskog, K.G., \& Sörbom, D. (1993). Lisrel 8: Structural equation modeling with the SIMPLIS command language. Chicago, IL: Scientific Software International. 
Malla, A.K., Norman, R.M.G., Williamson, P., Cortese, L., \& Diaz, F. (1993). The syndrome concept of schizophrenia. A factor analytic study. Schizophrenia Research, 10, 143-150.

Mellers, J.D.C., Sham, P., Jones, P.J., Toone, B.K., \& Murray, R.M. (1996). A factor analytic study of symptoms in acute schizophrenia. Acta Psychiatrica Scandinavica, 93, 92-98.

Murphy, J.A., Shevlin, M., Adamson, G., \& Houston, J. (2010). A population based analysis of sub-clinical psychosis and help-seeking behaviour. Schizophrenia Bulletin, 38, 360367.

Muthén, L.K., \& Muthén, B.O. (1998). Mplus user's guide (5th ed.). Los Angeles, CA: Muthen \& Muthen.

Psychosis

Nakaya, M., Suwa, H., Komahashi, T., \& Ohmori, K. (1999). Is schizophrenic symptomatology independent of the phase of the illness? Psychopathology, 32, 23-29.

Parker, S., \& Lewis, S. (2006). Identification of young people at risk of psychosis. Advances in Psychiatric Treatment, 12, 249-255. doi:10.1192/apt.12.4.249

Peralta, V., \& Cuesta, M.J. (2001). How many and which are the psychopathological dimensions in schizophrenia? Issues influencing their ascertainment. Schizophrenia Research, 49, 269-285.

Peters, E., Joseph, S., \& Garety, P. (1999). Measurement of delusional ideation in the normal population: Introducing the PDI (Peters, et al. Delusions Inventory). Schizophrenia Bulletin, 25, 553-576.

Poulton, R., Caspi, A., Moffitt, T.E., Cannon, M., Murray, R., \& Harrington, H. (2000). Children's self-reported psychotic symptoms and adult schizophreniform disorder: A 15year longitudinal study. Archives of General Psychiatry, 57, 1053-1058.

Reininghaus, U., Priebe, S., \& Bentall, R.P. (2012). Testing the psychopathology of psychosis: Evidence for a general psychosis dimension. Schizophrenia Bulletin, 39, 884895.

Schuldberg, D., Quinlan, D.M., Morgenstern, H., \& Glazer, W. (1990). Positive and negative symptoms in chronic psychiatric outpatients: Reliability, stability and factor structure. Journal of Consulting and Clinical Psychology, 2, 262-268.

Stefanis, N.C., Hanssen, M., Smirnis, N.K., Avramopoulos, D.A., Evdokimidis, I.K., Stefanis, C., ... Van Os, J. (2002). Evidence that three dimensions of psychosis have a distribution in the general population. Psychological Medicine, 32, 347-358.

Steiger, J.H. (1990). Structural model evaluation and modification: An interval estimation approach. Multivariate Behavioural Research, 25, 173-180.

Tanaka, J.S. (1987). How big is big enough? Sample size and goodness of fit in structural equation models with latent variables. Child Development, 58, 134-146.

Tucker, L.R., \& Lewis, C. (1973). The reliability coefficient for maximum likelihood factor analysis. Psychometrika, 38, 1-10.

Wigman, J.T., Vollebergh, W., Raaijmakers, Q., Iedema, J., van Dorsselaer, S., Ormel, J., ... Van Os, J. (2011). The structure of the extended psychosis phenotype in early adolescence - A cross-sample replication. Schizophrenia Bulletin, 37, 850-860. doi:10.1093/schbul/sbp154

Wing, J.K., Cooper, J.E., \& Sartorius, N. (1974). The description and classification of psychiatric symptoms. An instruction manual for the PSE and CATEGO systems. Cambridge: Cambridge University Press.

Yung, A.R., Buckby, J.A., Cotton, S.M., Cosgrave, E.M., Killackey, E.J., Stanford, C., ... McGorry, P.D. (2006). Psychotic-like experiences in nonpsychotic help-seekers: Associations with distress, depression, and disability. Schizophrenia Bulletin, 32, 352359.

Yung, A., Nelson, B., Baker, K., Buckby, J.A., Baksheev, G., \& Cosgrave, E.M. (2009). Psychotic-like experiences in a community sample of adolescents: Implications for the 
continuum model of psychosis and prediction of schizophrenia. The Australian and New Zealand Journal of Psychiatry, 43, 118-128. 